\title{
Simulation and Mathematical Description of Mechanical Shocks Caused by Brake Actuation on Electric Motors
}

\author{
David Schepers \\ University of Applied Sciences Ruhr-West \\ Mülheim an der Ruhr, Germany
}

Josef Börcsök

University of Kassel

Kassel, Germany

\author{
Lauri Bodenröder \\ University of Applied Sciences Ruhr-West \\ Mülheim an der Ruhr, Germany \\ Florian Rieger \\ SEW-EURODRIVE GmbH \& Co KG \\ Bruchsal, Germany
}

\begin{abstract}
When a brake engages or releases on an electric motor, a mechanical shock is generated. These so-called brake shocks propagate across the motor housing and the motor shaft, affecting safety relevant mechanical and electronic components. The nature of the interference may be irreversible, i.e. mechanical damage, or reversible, e.g. interference of signal measurement or data transmission. Especially component failures or faulty signal values on rotary encoders are undesirable from a safety point of view. Current shock testing procedures are insufficient to simulate real brake shock characteristics and to identify valid shock limits regarding these shocks. In the first part of this paper, the characteristics of brake shocks are presented and compared to pyroshocks with similar characteristics. Furthermore, it shows that the Pseudo-Velocity Shock Response Spectrum (PVSRS) appears to be the best mathematical method to describe the severity of brake shocks with respect to their potential of damaging encoder components or influencing electrical signals. In the second part a testing machine will be introduced, which is able to generate mechanical shocks with comparable characteristics of real mechanical brake shocks for up to several million cycles. During further research, endurance tests shall be performed with the machine to determine the resilience of safety-related components against mechanical brake shocks. The long-term goal is to define scientifically confirmed test criteria for a standardized shock testing procedure to be applied on safety-related components on electric motors. It is intended to include this testing procedure in an international safety-related standard, like IEC 61800-5-3.
\end{abstract}

Keywords: Systems Theory, Applied Systems Theory, Signals, Applied Signal Processing, Functional safety, Mechanical shock, Reliability, Electric Motors, rotary encoder

\section{INTRODUCTION}

Mechanical influences on safety-relevant components currently receive little attention in the field of functional safety. For example, common normative requirements for vibration and shock testing procedures are not able to reproduce the characteristics of real mechanical brake shocks. According to the current state of science, brake shocks are not sufficiently understood. Particularly, it has to be investigated in how far brake shocks in continuous operation with high braking cycles have the potential to cause critical failures in safety-relevant components. In order to improve the general understanding of mechanical brake shocks and to derive adequate testing procedures, this paper will focus on the simulation and analysis of mechanical shocks caused by brake actuation on electric motors. First, the typical characteristics of brake shocks are presented and mathematical criteria to evaluate the severity of brake shocks are defined. Subsequently a testing machine, which is suitable for conducting endurance tests on rotary encoders by simulating real characteristics of mechanical brake shocks, is presented. The simulated brake shocks are compared to real brake shocks, which were directly measured at the motor shaft.

\section{BRAKE SHOCK CHARACTERISTICS}

Brake shocks, which are generated by brake application, propagate across the motor housing and the motor shaft, as shown in Fig. 1, affecting safety-relevant mechanical and electronic components. These influences may be irreversible, i.e. mechanical damage, or reversible, e.g. interference of signal measurement or data transmission. Especially encoders, which are attached to the end of the motor shaft for detection of speed and position, may suffer these degradations. This might have a significant influence on safety, as uncontrolled motor movement might lead to harmful situations [1]

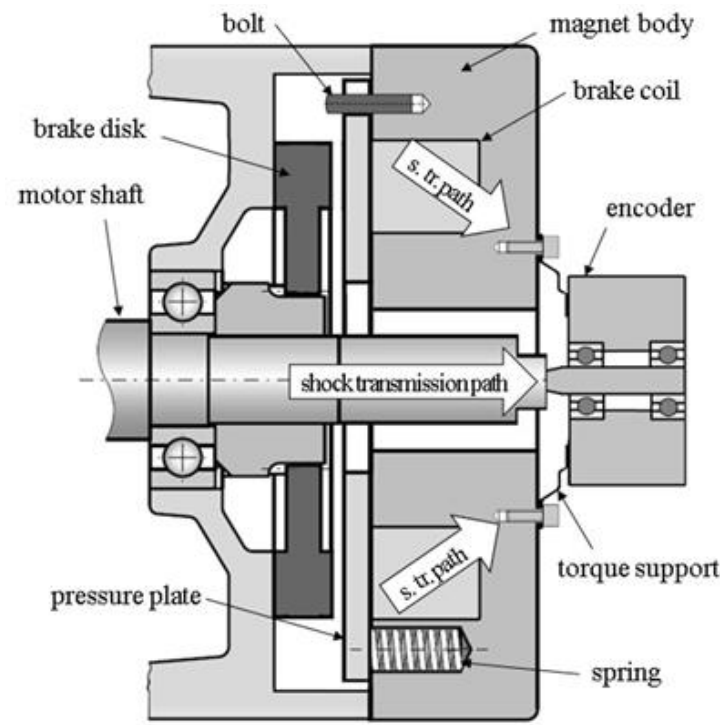

Fig. 1 Shock propagation on electric motors [1]

Simple and robust data acquisition can be carried out by means of acceleration sensors, as measurement of deflection is imprecise due to high frequencies and low amplitude. 
Acceleration sensors are available, whose limiting frequency is high enough not to influence data acquisition. As can be seen in Fig 1, shocks may propagate via stator (magnet body) or via rotor (motor shaft). For this reason, measurements may be carried out at the motor stator or at the motor shaft (which is more difficult to realize due to motor movement) in order to investigate the severity of the shock, which is transmitted to the encoder. Another possibility is to take measurements at the housing of the encoder, where the response spectrum of the encoder housing can be recorded [1]. Measurements at the encoder housing may deliver information about specific resonance frequencies and damping characteristics of the housing. In this paper, however, measurements will be presented which were acquired at the end of the motor shaft, as measurements have shown that this is the main shock transmission path. By measuring at the end of the motor shaft, it shall be possible to evaluate the severity of transmitted brake shocks for different motor/brake configurations independently of the housing characteristics.

Fig. 2 shows a typical high intensity brake shock, which was measured directly at the end of the motor shaft, while the brake engaged at around $1500 \mathrm{rpm}$. The approximate shock duration is 10 to $15 \mathrm{~ms}$ with peak amplitudes of up to $500 \mathrm{~g}$.

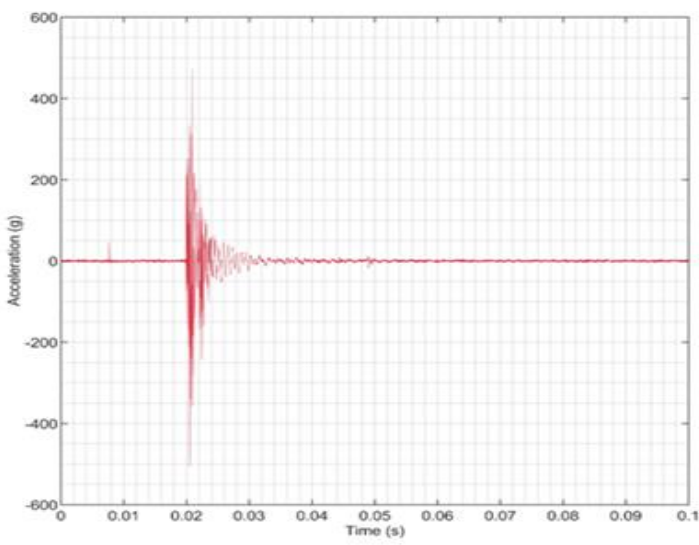

Fig. 2 Real brake shock (measured on motor shaft)

In Fig. 3 a typical pyroshock is depicted. Comparing both time histories of the presented shocks, strong similarities in their characteristics regarding duration, shape and amplitude can be observed.

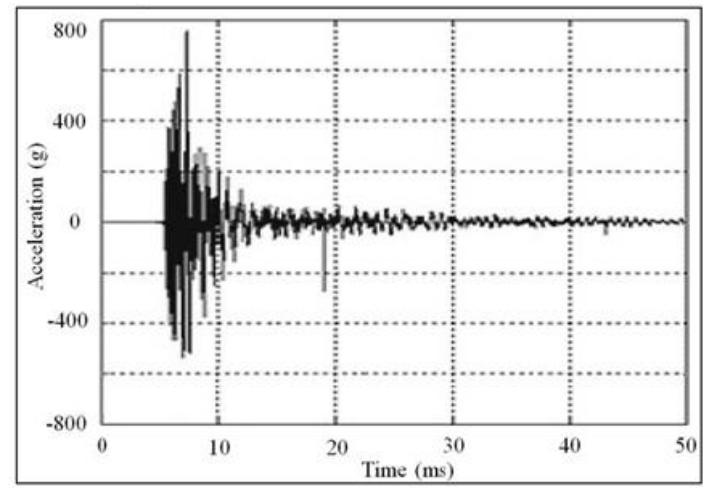

Fig. 3 Far-field pyroshock (example) [2]
Due to these similarities between brake shocks and pyroshocks, well established methods of data analysis for pyroshocks can be applied to classify brake shocks [1].

\section{SEVERITY CLASSIFICATION OF BRAKE SHOCKS}

The severity of a brake shock depends on several factors. Firstly, the characteristics of the brake itself, such as the braking force and the size of the air gap between the brake disc and the pressure plate. The larger this air gap or braking force is, the greater the severity of the shock. Secondly, the rotation velocity of the motor shaft. Brake application at standstill is less severe than with shaft rotation. However, maximum shock severity caused by spring-applied brakes is reached in the range of 150 to 500 rotations per minute and no further significant increase in shock severity can be observed up to 6000 rotations per minute [1]. In order to compare the severity of different shocks, it is not useful to compare the time series of the measured acceleration, velocity or displacement of the shocks. This is because on the one hand, even completely different mechanical shocks can show similar damage potential and on the other hand, the resonance vibration of the shock environment is significantly longer than the excitation by the shock itself, whereby the excitation of the eigenmodes of the components is particularly responsible for the damage of components in the shock environment [3]. Therefore, a consideration of the shock response spectrum is more revealing. It has been shown that using Pseudo-Velocity Shock Response Spectra (PVSRS) appears to be the best mathematical method to describe the severity of mechanical shocks regarding their damage potential, because pseudo-velocity is proportional to mechanical stress and pseudo-velocity shows the energy content of the shock $[4,5]$. The PVSRS is determined by the maximum pseudo-velocity of a set of hypothetical singledegree-of-freedom oscillators with different natural frequencies based on the measured acceleration over the time domain of the shock and then plotted over the frequency domain [6]. It is usually plotted on fourcoordinate-paper, as illustrated in Fig 4. This mode of plotting allows to display the four relevant parameters in the same diagram: Frequency, pseudo-velocity, deflection and acceleration.

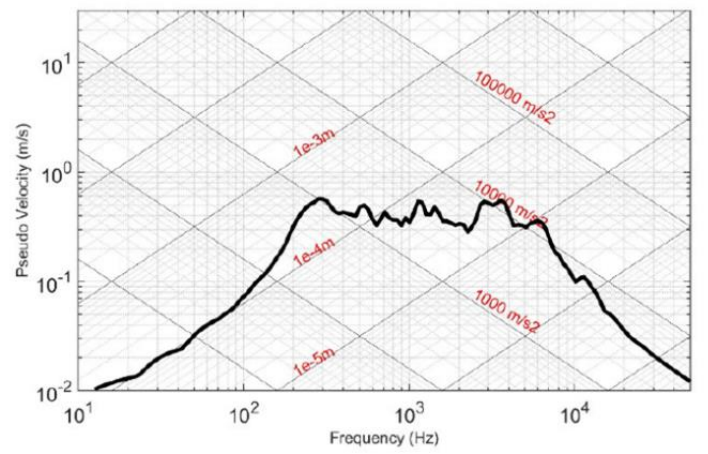

Fig. 4 Frequency, pseudo-velocity, deflection and acceleration plotted on four-coordinate-paper

\section{TESTING MACHINE FOR BRAKE SHOCK SIMULATION}

Current shock testing procedures are insufficient to simulate real brake shock characteristics and to determine valid shock limits in order to avoid damages on safety relevant components caused by these shocks. For instance, 
most rotary encoders are currently shock-tested with halfsine excitations in accordance to EN 60068-2-27. However, characteristics of real mechanical brake shocks cannot be reproduced by half-sine excitations, as brake shocks cover a wide range of frequencies. In addition to this, shock testing procedures applying half-sine excitations only cover a low number of cycles, whereas brake shocks can occur millions of times, depending on the application. For these reasons, it is necessary to define a reproducible method for determination of encoder robustness against brake shocks. Using PVSRS to classify brake shocks allows to evaluate the reproducibility of a mechanical shock because it clearly displays the pseudo-velocity in frequency domain. As the potential for damages correlates with the pseudo-velocity, similar PVSRS curves should have similar potential for damages [4]. Therefore, by simulating PVSRS curves with similar characteristics compared to real brake shocks for several millions of cycles, it is possible to apply these endurance tests to evaluate the robustness of rotary encoders against brake shocks.

A testing machine for the simulation of the severity of brake shocks was developed in the laboratories of SEW and is depicted in Fig 5. This testing machine is particularly suitable for simulating high frequency and multi axis shock loads which can be expected when motor brakes engage. The encoder under test is connected to the shaft and the stator of the testing machine. It can be tested under normal operation conditions, i.e. when the shaft is rotating with constant speed and encoder signals are monitored. During this operation, shock excitation is realized by the impact of actuators on the shaft and stator of the machine. In Fig. 5, two rotary actuators acting on the shaft and a further linear actuator acting on the stator of the testing machine are being shown as an example. Other actuator configurations for shock excitation may be realized in order to simulate different shock loads.

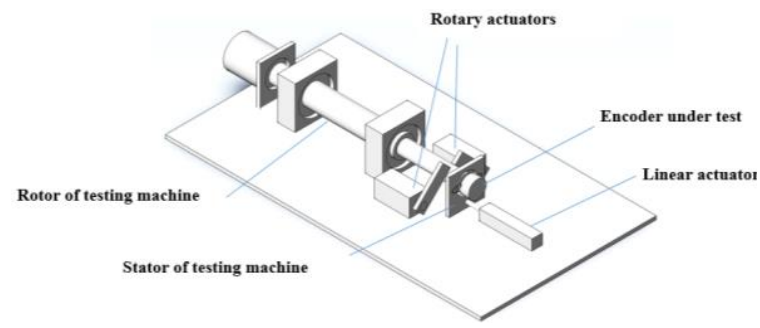

Fig. 5 Schematic representation of testing machine for brake shock simulation

To achieve the desired test shock load, several parameters of different parts of the testing machine can be varied. These include natural frequencies, damping and mass of the shaft and the stator of the testing machine; the position, direction and number of actuators as well as the velocity, mass and material of the impactors.

In Fig. 6, the measured acceleration of a mechanical shock generated by the testing machine, in red, is compared to the measured acceleration of a real brake shock, in blue. Both signals were directly measured at the end of the motor shaft and filtered with a $100 \mathrm{~Hz}$ to $10,000 \mathrm{~Hz}$ FFT bandpass filter. The acceleration of the shocks show clear similarities in amplitude in positive direction. However, the amplitude of the simulated shock is less intense in negative direction. Furthermore, the shape of both shocks is similar until some low-intense oscillation occurs in the simulated shock between $35 \mathrm{~ms}$ to $50 \mathrm{~ms}$.

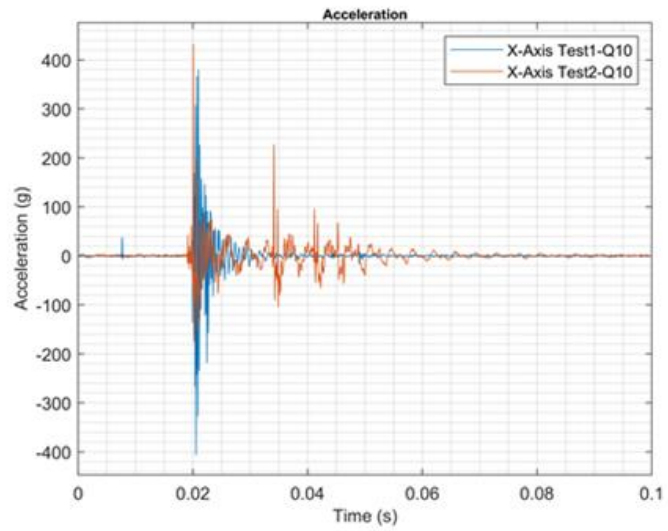

Fig. 6 Brake shock (blue) and testing machine shock (red)

Due to this oscillation, the simulated shock contains higher accelerations in the lower frequency range, which is revealed by the calculated FFT (see Fig. 7)

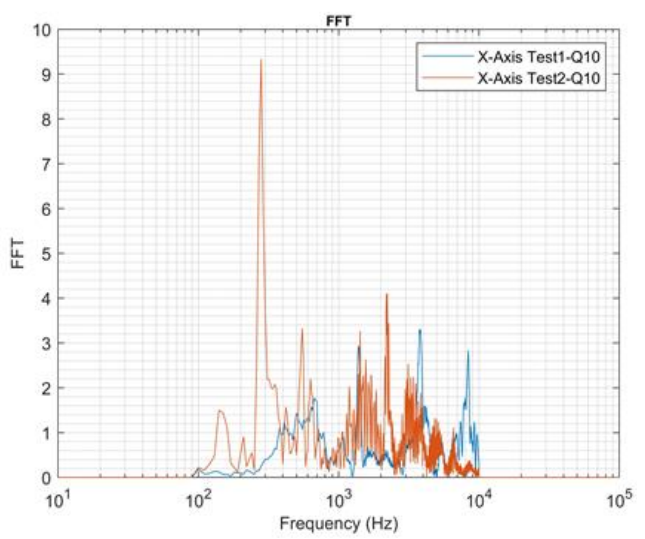

Fig. 7 FFT of brake shock (blue) and of testing machine shock (red)

The PVSRS was calculated for both shocks in accordance with ISO 18431-4 and a Q factor of ten (Fig. 8). Despite the differences in the acceleration time histories of the shocks, the PVSRS show strong similarities especially in the high frequency region from $500 \mathrm{~Hz}$ to $10,000 \mathrm{~Hz}$. Only in the frequency region below $500 \mathrm{~Hz}$, the simulated shock shows significantly higher damage potential. However, the eigenmodes of rotary encoders (excluding the torque support) are usually not within this low-frequency range and therefore will not be stimulated by these shock frequencies. That means, it can be expected that the damaging influence of vibration frequencies below $500 \mathrm{~Hz}$ will be low. 


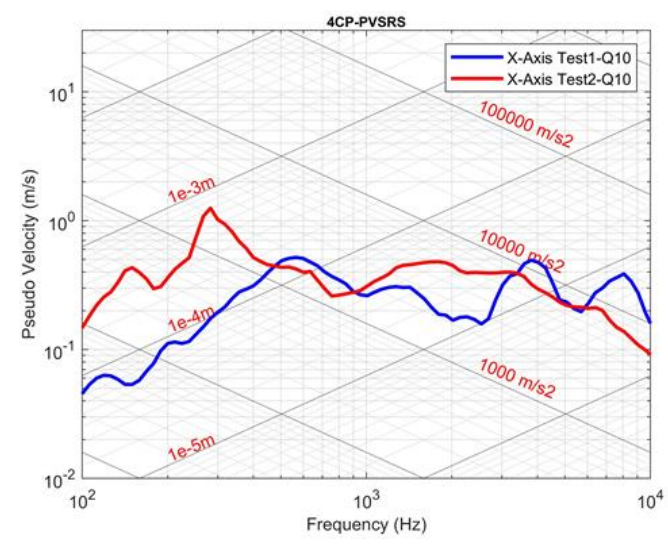

Fig. 8 Calculated PVSRS plotted in a four-coordinate-diagram

\section{CONCLUSION}

In the first part of the paper, the characteristics of brake shocks were described and it was shown that they are comparable to pyroshocks, because of strong similarities in duration, shape and amplitude. It was also explained why the Pseudo-Velocity Shock Response Spectrum (PVSRS) is a suitable criterion to describe the severity of mechanical brake shocks. In the second part, a testing machine was introduced and an example measurement of a mechanical brake shock generated by the testing machine was presented and analyzed. Although the simulated shock showed differences in comparison to a shock caused by brake actuation on an electric motor, in the most relevant high frequency range both shocks showed comparable severity characteristics. Further modifications on the testing machine are necessary to improve the low frequency characteristics of the generated shock and to match the high frequency characteristics more precisely. In future research, endurance tests shall be performed with the machine to determine the resilience of safety-related components against mechanical brake shocks. The long-term goal is to define scientifically confirmed test criteria for a standardized shock testing procedure to be applied on safety-related components on electric motors. It is intended to include this testing procedure in an international safety-related standard, like IEC 61800-5-3.

\section{REFERENCES}

[1] F. Rieger, B. Hagemann, Mechanical shocks caused by brakes on electric motors In Innovative Small Drives and Micro-Motor Systems; 11th GMM/ETG-Symposium. Saarbrücken, Germany: VDE, 2017.

[2] NASA-STD-7003A: Pyroshock Test Criteria, 2011.

[3] ECSS, Space Engineering - Mechanical shock design and verification handbook, Noordwijk, Netherlands: ESA Requirements and Standards Division, 2015.

[4] H.A. Gaberson, Pseudo Velocity Shock Spectrum Rules for Analysis of Mechanical Shock, 2007.

[5] H.A. Gaberson, Shock Severity Estimation. Sound \& Vibration January 2015 - 45th Anniversary Issue, 2012, pp. 12-19.

[6] ISO 18431-4:2007. Mechanical vibration and shock - Signal processing. Part 4: Shock-response spectrum analysis

[7] V. I. Bateman, H. Himelblau, and R. Merritt, Validation of Pyroshock Data, Sound \& Vibration - March 2012 - Dynamic Testing, 2012, pp. 6-11.

[8] M.R. Grundvig, H. Himelblau, A.G. Piersol, and J.H. Wise, Handbook for Dynamic Data Acquisition and Analysis, Mount Prospect: Institute of Environmental Sciences, 1994.

[9] A.G. Piersol, Pyroshock Data Acquisition and Analysis for U/RGM109D Payload Cover Ejection Tests, China Lake: Naval Weapons Center, 1998.

\section{Creative Commons Attribution License 4.0 (Attribution 4.0 International, CC BY 4.0)}

This article is published under the terms of the Creative Commons Attribution License 4.0

https://creativecommons.org/licenses/by/4.0/deed.en_US 